\title{
Systematic studies of Canthecona furcellata (Wolf 1851) (Hemiptera: Pentatomidae: Asopinae) from Khairpur, Sindh, Pakistan
}

\author{
Shabana Mangi ${ }^{1}$, Abdul Manan Shaikh ${ }^{1}$, Waheed Ali Panhwar ${ }^{*}$, Wali \\ $\mathrm{Khan}^{2}$ and Muzafar Shah ${ }^{3}$ \\ 1. Department of Zoology, Shah Abdul Latif University Khairpur Mirs Sindh-Pakistan \\ 2. Department of Zoology, University of Malakand, KPK-Pakistan \\ 3. Department of Zoology, University of Swat, KPK-Pakistan \\ *Corresponding author's email: waheed.panhwar@salu.edu.pk \\ Citation \\ Shabana Mangi Abdul Manan Shaikh, Waheed Ali Panhwar, Wali Khan, Muzafar Shah. Systematic studies of \\ Canthecona furcellata (Wolf 1851) (Hemiptera: Pentatomidae: Asopinae) from Khairpur, Sindh, Pakistan. Pure and \\ Applied Biology. Vol. 10, Issue 3, pp928-934. http://dx.doi.org/10.19045/bspab.2021.100096
}

Received: 29/03/2020 Revised: 19/12/2020

Accepted: 21/12/2020

Online First: 02/01/2021

\section{Abstract}

During Pentatomid bugs studies of Canthecona furcellata (Wolf 1851) was collected from the Sobhodero, Gambat, Faizgang Taluka of district Khairpur is a predator of various larva of Lepidoptera, Coleoptera and Hymenoptera of pests, the taxonomical features of current specimens differ from the body measurements coloration, male, female genitalial structures pygophore, aedeagus, parameres, different taxonomical characteristics, dorsolateral lobe of pygophore, narrow, short stem of paramere, female genitalial features $1^{\text {st }}$ gonocoxae triangular in, $9^{\text {th }}$ para tergites elongates, spermathecal bulb elliptical, this species is a new recorded from the district Khairpur.

Keywords: Asopinae; Genitalia; Khairpur; Morphology; New record

\section{Introduction}

Asopinae predatory stink bugs belong to order Hemiptera, they are feed on the different larva of Coleoptera, Hymenoptera and Lepidoptera and larva pest on different crops, their role as important biological pest control agents has been manipulated in the field, (Schuh and Slater 1995) they have 300 species, 60 genera they are differs from other Pentatomid within subfamilies mainly having a predaceous feeding habits, they are scattered at worldwide [1-9]. The genus Canthecona was belong to subfamily
Asopinae commonly called predatory stink bugs, contains the new species first reported from Pakistan along with male, female genitalia, scent gland, the Canthecona furcellata (Wolff 1851) is insect-pest species , organization is depend on ecological principles, integration, formation and control on different elements of action of an management system of insect-pest, due to protect the chemical toxicity arising out by the use of insecticides, examined and achieve the benefits from these natural enemies for the control of pest species, identification on 
the basis of body coloration, mouthparts, antennae, labium and male, female genitalia, the current thoughts of insect-pest controlling is based on environmental ethics and included the incorporation and combinations of various mechanisms, resistors strategies into an insect-pest management system.

In opinion of chemical threats rising out of indiscriminate use of insecticides, examinations and manipulation of natural enemies for the resistor of recognized important pest becomes essential. Many biotic agents control the population [9-11]. The Canthecona furcellata (Wolff 1851) reported from the Karachi in Sindh, Khyber Pukhtainkhuwa, Baluchistan, Islamabad, Changmanga, Rawalpindi in Punjab, Hazara in Peshawar, Balakot in KPK, Sylhet in East Bengal Burma, Bahamas, Java, Ceylon and various parts of Indo-Pak, the genus [12-15]. Canthecona furcellata described by (Wolff, 1851), predators on various larva of Lepidoptera, Hymenoptera and Coleoptera, present here we have revised this species first time recorded from the Khairpur District and its adjoining areas Sind Pakistan, the explanation of this species is based not only the morphological characteristics but we too explained the illustrate the male and female genitalia.

\section{Materials and Methods}

The samples were together from the various location of the district Khairpur, (from March 2014 to October 2016), kept in bottles, paralyses with chloroform, then preserved in timber boxes, naphthalene balls be used for protection [16]. The abdomen of male specimens was dipped into warm water for about (2 to 3 minutes) after that male genitalia (pygophore) remove from the abdomen with the help of fine tapered forceps. The internal structures of male (pygophore) was heated in $10 \% \mathrm{KOH}$ for $25-$ 30 minutes After remove $(\mathrm{KOH})$ with tap water, the parameres (clasper) and aedeagus were extracts from pygophore and out the aedeagus were inflated with the support of pointed forceps below the dissecting microscope was reported [17]. Observing the structures of female, the stuck female sample were dipped into warm water for (2 to 3 minutes), the abdomen was extract and boiled in $10 \% \mathrm{KOH}$ for 15 minutes, and the spermtheca was visibly by partially extract the tergite as explained [18]. Whole measurements occurred through the millimeters. The measurements of different body portions were taken with the help of an ocular micro, Images were traced with turning pointer upon butter paper beneath the dissecting microscope [18] at Shah Abdul Latif University Khairpur.

\section{Results}

Number of specimen collected: 493

Number of specimen recognized: 178

Area: District Khairpur, Sindh, Pakistan

Family: Pentatomidae

Subfamily: Asopinae

Tribe: Asopini

Genus: Canthecona Amyot et Serville

Species: Canthecona furcellata (Wolff 1851) (Table 1 \& Fig. 1)

\section{Body coloration}

Body grey, light brown body covered with minor punctuation present, without head, a black in color, head covered with yellowish lines, color of eyes black, pink ocelli, humeral angle black yellowish to brown, segments of antennae 5th,4th and 3rd brown, densely brown pronotum, scutellum intermediate between black and white, ventral margins of body, legs color grey, deeply brown wings, tibia dark brown and femora light brown .

\section{Head}

Head length $(1.2 \mathrm{~mm})$, width $(1.8 \mathrm{~mm})$, broader than longer, paraclypeus tip circular, tapered, elongated than clypeus, antennae $3^{\text {rd }}$ section elongated than $2^{\text {nd }}$ section, $1^{\text {st }}$ section measurements is $(0.3 \mathrm{~mm}), 2^{\text {nd }}$ section measurements $(1.33 \mathrm{~mm}), \quad 3^{\text {rd }}$ section 
measurements $\quad(1.35 \mathrm{~mm}), \quad 4^{\text {th }}$ section measurements $\quad(1.5 \mathrm{~mm}), \quad 5^{\text {th }}$ section measurements $(1.4 \mathrm{~mm})$, antennal formula $(1<2<3<5<4)$, rostrum measurement $1^{\text {st }}$ section $(1 \mathrm{~mm}), 2^{\text {nd }}$ section $(1.4 \mathrm{~mm}), 3^{\text {rd }}$ section $(1.3 \mathrm{~mm}), 4^{\text {th }}$ section $(9 \mathrm{~mm})$, labial formula $(3<2<1=4)$, rostrum reached to hind coxae, measurements between the anterior anteocular $(7 \mathrm{~mm})$, posterior anteocular $(.2 \mathrm{~mm})$ and interocular measurements $(9 \mathrm{~mm})$.

\section{Thorax}

Prontal angles pointed and have bifid spine, pronotum length $(1.3 \mathrm{~mm})$ and width $(4 \mathrm{~mm})$, scutellum length $(2.2 \mathrm{~mm})$, width $(1.8 \mathrm{~mm})$, base pointed and broader at the anterior region

\section{Abdomen}

In female specimens have a $7^{\text {th }}$ abdominal section of Ventroposterior margins of sternum broad, concave, but in male specimen usually have a convex, total body length $(9.4 \mathrm{~mm})$.

\section{Female genitalia}

Concial first gonocoxae, innermost sides parallel, external sides of posterior sides usually have a convex, $9^{\text {th }}$ lengthened paratergites reaching upto posterior sides of $8^{\text {th }}$ paratergites, $2^{\text {nd }}$ gonocoxae fused with posterior sides, Spermathecal bulb have a lengthened oval in shape, middle dilation triangular in shape.

\section{Male genitalia}

Aedeagus broader than longer, Pygophore of dorsolateral lobe distinctly thin, broader, median sides of dorsoposterior convex, dorsolateral sides are usually in concave in shape, small stem of paramere, blade broader anterior region concave and posterior region convex, base bigonal, thin, conical at tip, two section of conjunctival appendages, and a have a pair pouch like structures, dorso median broader inward.

\section{Discussion}

Genus Canthecona Amyot et Serville commonly known as predatory stink bugs found in Pakistan, Bengal, India, Burma, Java, Bhamo, the predatory found in March to September month of the year, morphological characteristics was given by Ahmad and McPherson [19]. There is no recently work available on the morphology and male, female genitalia, here we described the species on the basis of morphology and genitalia features body grey, brown pale body covered with minute deeply punctuations present, without head, a black in color, head covered with yellowish lines, color of eyes black, humeral angles of pronotum tapered tip bifid spine, the length of pronotum shorter than width, the length of pronotum, Conical first gonocoxae, innermost sides parallel, external sides of posterior sides usually have a convex, $9^{\text {th }}$ lengthened paratergites reaching upto posterior sides of $8^{\text {th }}$ paratergites, $2^{\text {nd }}$ gonocoxae fused with posterior sides, Spermathecal bulb have a lengthened oval in shape, middle dilation triangular in shape, Aedeagus broader than longer, Pygophore of dorsolateral lobe distinctly thin, broader, median sides of dorsoposterior convex, dorsolateral sides are usually in concave in shape, small stem of paramere, blade broad at anterior region concave. Canthecona furcellata (Wolff 1851) first time collected from the various localities of district of Khairpur. So therefore it is a new record of this species in Khairpur district in province of Sind Pakistan. 
Table 1. Showing a comparative morphological measurement and features of Canthecona furcellata (Wolf 1851)

\begin{tabular}{|c|c|c|c|c|c|c|c|}
\hline Body parts & Current species & $\begin{array}{c}\text { C. neotibialis }(1985) \\
15.7+15.6(\mathrm{~mm})\end{array}$ & $\begin{array}{c}\text { C. parva }(1978) \\
11.8+12.1(\mathrm{~mm})\end{array}$ & $\begin{array}{c}\text { C. populusai }(1985) \\
10.1+12.1(\mathrm{~mm})\end{array}$ & $\begin{array}{c}\text { C. binotata }(1978) \\
15+11.7(\mathrm{~mm})\end{array}$ & $\begin{array}{c}\text { C. ornatula }(1909) \\
14+15(\mathrm{~mm})\end{array}$ & $\begin{array}{c}\text { C. robuata } \\
11+14(\mathrm{~mm})\end{array}$ \\
\hline $\begin{array}{l}\text { Coloration and } \\
\text { body size }\end{array}$ & $\begin{array}{l}\text { Grey to pale } \\
\text { brown and } \\
\text { elongated, slightly } \\
\text { broaded }\end{array}$ & $\begin{array}{l}\text { Body dark brown and } \\
\text { elongated size }\end{array}$ & Dark ochraceous & $\begin{array}{l}\text { Lacteous to redish } \\
\text { brown }\end{array}$ & Brown to blackish & Green, metallic & $\begin{array}{l}\text { Lacteous to } \\
\text { brown }\end{array}$ \\
\hline Head & $\begin{array}{l}\text { paraclypeus } \\
\text { round, narrow } \\
\text { longer than } \\
\text { clypeus }\end{array}$ & $\begin{array}{l}\text { paraclypeus convex, } \\
\text { wide } \\
\text { than clypeus }\end{array}$ & $\begin{array}{l}\text { Lengthened, } \\
\text { wider paraclypei } \\
\text { flat, clypeus } \\
\text { shorter }\end{array}$ & $\begin{array}{l}\text { Lengthened as well } \\
\text { as wided paraclypei } \\
\text { narrow }\end{array}$ & $\begin{array}{l}\text { Head covered with } \\
\text { punctured }\end{array}$ & $\begin{array}{l}\text { Convex, elongated, } \\
\text { width between the } \\
\text { outer sides of eyes }\end{array}$ & $\begin{array}{l}\text { Concave } \\
\text { lengthened }\end{array}$ \\
\hline Antennae(mm) & $\begin{array}{l}\text { lengthened } \\
\mathrm{I}=0.3, \mathrm{ii}=1.33, \\
\mathrm{iii}=1.35, \mathrm{iv}=1.5, \\
\mathrm{v}=1.4 .\end{array}$ & $\begin{array}{l}\text { Lengthened } \\
\mathrm{I}=0.4, \mathrm{ii}=1,2, \mathrm{iii}=1,36, \mathrm{i} \\
\mathrm{v}=1.6, \mathrm{v}=1.5\end{array}$ & $\begin{array}{l}\text { Mutilated } \\
\mathrm{I}=0.3, \mathrm{ii}=1.3, \mathrm{iii}=1 . \\
3, \mathrm{iv}=1.6, \mathrm{v}=\text { damag } \\
\text { ed }\end{array}$ & $\begin{array}{l}\text { Elongated } \\
\mathrm{I}=0.3, \mathrm{ii}=1.1, \mathrm{iii}=1.1, \mathrm{iv} \\
=1.3, \mathrm{v}=1.1\end{array}$ & $\begin{array}{l}\text { lengthened } \\
\mathrm{I}=0.2, \mathrm{ii}=1.3, \mathrm{iii}=1.4 \\
\mathrm{iv}=1.6, \mathrm{v}=0.4\end{array}$ & $\begin{array}{l}3^{\text {rd }}, 4^{\text {th }} \\
\text { sectionsreaced to } \\
\text { half of eyes, } 5^{\text {th }} \\
\text { mutilated }\end{array}$ & $\begin{array}{l}\text { Lengthened } \\
\mathrm{I}=0.3, \mathrm{ii}=0.12, \mathrm{iii} \\
=0.3, \mathrm{iv}=1, \mathrm{v}=0.1\end{array}$ \\
\hline $\begin{array}{l}\text { Antennal } \\
\text { formula }\end{array}$ & $1<2<3<5<4$ & $1<2<4<35<1$ & $2<1<4, \mathrm{ii}=\mathrm{iii}$ & $1<2=3<5<4$ & $\mathrm{Ii}=\mathrm{ii}, \mathrm{iv}<\mathrm{v}<\mathrm{i}$ & $3=4,2=4$ & $1=3,2<5$, \\
\hline Labium(mm) & $\begin{array}{l}\mathrm{I}=1, \mathrm{ii}=1.4, \mathrm{iii}=1.3, \\
\mathrm{iv}=9\end{array}$ & $\begin{array}{l}\mathrm{I}=0.4, \mathrm{ii}=1.8, \mathrm{iii}=1.6, \mathrm{iv} \\
=1.3\end{array}$ & $\begin{array}{l}\mathrm{I}=1.1, \mathrm{ii}=1.3, \mathrm{iii}=1 . \\
1, \mathrm{iv}=0.9\end{array}$ & $\begin{array}{l}\mathrm{I}=0.9, \mathrm{ii}=1.2, \mathrm{iii}=1.0, \mathrm{iv} \\
=0.9\end{array}$ & $\begin{array}{l}\mathrm{I}=0.3, \mathrm{ii}=1.5, \mathrm{iii}=1.1, \\
\mathrm{iv}=1\end{array}$ & 4 sectioned & Mutilated \\
\hline Labial formula & $3<2<1=4$ & $4<1<3<2$ & $4<3,1<2$ & $1=4<3<2$ & $2<3<4<1$ & Not & Not \\
\hline Thorax & flat & Wide & Deflected & Broaded & wider & Wider than longer & Broaded \\
\hline $\begin{array}{l}\text { Pronotum } \\
(\mathrm{mm})\end{array}$ & $\begin{array}{l}\text { Wider than longer } \\
\text { and prontal angles } \\
\text { pointed } \\
1.3\end{array}$ & $\begin{array}{l}\text { Distinctly wider than } \\
\text { longer } \\
2.6\end{array}$ & $\begin{array}{l}\text { Broader, prontal } \\
\text { angles slightly } \\
\text { tapered } \\
2.8\end{array}$ & $\begin{array}{l}\text { Broader than longer, } \\
\text { acute tubercles } \\
2.6\end{array}$ & $\begin{array}{l}\text { Wide, flat, sinuate } \\
2.4\end{array}$ & $\begin{array}{l}\text { Longer than wider, } \\
\text { fully punctate } \\
1.6\end{array}$ & $\begin{array}{l}\text { Anterior } \\
\text { narrow, broaded } \\
\text { at posterior } \\
1.2\end{array}$ \\
\hline Width & 4 & 6.6 & 7.3 & 6.7 & 5.4 & 4 & 4 \\
\hline Scutellum & $\begin{array}{l}\text { Anterior margins } \\
\text { wider than } \\
\text { posterior tip } \\
\text { round } \\
2.2\end{array}$ & $\begin{array}{l}\text { Anterior margins } \\
\text { slightly broader tip } \\
\text { slightly pointed } \\
4.0\end{array}$ & Longer than wider & $\begin{array}{l}\text { Slightly longer than } \\
\text { wider at base, apical } \\
\text { lobe circular } \\
3.9\end{array}$ & $\begin{array}{l}\text { Wider, prontal } \\
\text { angles obscures, } \\
\text { thick, with black } \\
\text { spine } \\
4.2\end{array}$ & $\begin{array}{l}\text { Covered with finely } \\
\text { punctuation }\end{array}$ & $\begin{array}{l}\text { With orange to } \\
\text { yellow spot at } \\
\text { basal angle } \\
11\end{array}$ \\
\hline Width & 1.8 & 3.3 & 3.4 & 3.3 & 3.1 & 2.5 & 9 \\
\hline Abdomen & $\begin{array}{l}\text { Convex in male\& } \\
\text { concave in female }\end{array}$ & $\begin{array}{l}\text { Ventrolateral sides } \\
\text { narrow }\end{array}$ & $\begin{array}{l}\text { Ventrolateral } \\
\text { margins minutely } \\
\text { narrow }\end{array}$ & $\begin{array}{l}\text { Elytra narrow, lateral } \\
\text { margins thick }\end{array}$ & $\begin{array}{l}\text { Margins with green } \\
\text { punctures }\end{array}$ & Slightly convex & $\begin{array}{l}\text { Narrow at } \\
\text { anterior, } \\
\text { broaded at } \\
\text { posterior }\end{array}$ \\
\hline $\begin{array}{l}\text { Male genitalia } \\
\text { Pygophore }\end{array}$ & Wider than longer & $\begin{array}{l}\text { Narrow, concave, } \\
\text { lobe like }\end{array}$ & $\begin{array}{l}\text { Dorsolateral lobe } \\
\text { prominent, } \\
\text { widely, concave }\end{array}$ & $\begin{array}{l}\text { Lobes narrow } \\
\text { circular, concave, }\end{array}$ & $\begin{array}{l}\text { Dorsolateral lobe } \\
\text { prominent, widely, } \\
\text { concave }\end{array}$ & Broader than longer & $\begin{array}{l}\text { Wider than } \\
\text { longer }\end{array}$ \\
\hline
\end{tabular}




\begin{tabular}{|c|c|c|c|c|c|c|c|}
\hline Aedeagus & Wide, convex & Trunk like lengthened & & $\begin{array}{l}\text { Lengthened, base } \\
\text { convex, tip concave }\end{array}$ & elongated & Inflated aedeagus & $\begin{array}{l}\text { Anterior } \\
\text { margins are } \\
\text { wider }\end{array}$ \\
\hline Paramere & Sickle shaped & $\begin{array}{l}\text { Stem short, blade } \\
\text { wide }\end{array}$ & $\begin{array}{l}\text { Short, stem ,blade } \\
\text { wide, tip narrow }\end{array}$ & $\begin{array}{l}\text { With small stem } \\
\text {,blade lengthened }\end{array}$ & Blade long & Slightly rounded & Slightly tapered \\
\hline $\begin{array}{l}\text { Female } \\
\text { genitalia }\end{array}$ & $\begin{array}{l}9^{\text {th }} \text { paratergies } \\
\text { elongates } \\
\& \text { Spermathecal } \\
\text { bulb elliptical }\end{array}$ & $\begin{array}{l}1^{\text {st }} \text { gonocoxae } \\
\text { concial,spermthecal } \\
\text { bulb narrow }\end{array}$ & $\begin{array}{l}1^{\text {st }} \text { gonocoxae } \\
\text { triangular, } 9^{\text {th }} \\
\text { paratergies } \\
\text { lengthened }\end{array}$ & $\begin{array}{l}1^{\text {st }} \text { gonocoxae } \\
\text { concial, inner sides } \\
\text { sinuate, posterior } \\
\text { convex }\end{array}$ & $\begin{array}{l}1^{\text {st }} \text { gonocoxae } \\
\text { triangular, } 9^{\text {th }} \\
\text { paratergies } \\
\text { lengthened }\end{array}$ & $\begin{array}{l}1^{\text {st }} \text { gonocoxae wider } \\
\text { at base rounded at } \\
\text { tip }\end{array}$ & $\begin{array}{l}9^{\text {th }} \text { paratergies } \\
\text { slightly concial }\end{array}$ \\
\hline Host & $\begin{array}{l}\text { Grass, herbs, } \\
\text { shurbs }\end{array}$ & Dalbergia sissoo & Uthesia pulchela & Populous nigra & Naga hills & $\begin{array}{l}\text { Himalayan foot } \\
\text { hills }\end{array}$ & $\begin{array}{l}\text { Dalbergia } \\
\text { sissoo }\end{array}$ \\
\hline Locality & $\begin{array}{l}\text { Sobhodero } \\
\text { (Khairpur) }\end{array}$ & Islamabad, Punjab & Bangladesh & Punjab & India & India & Ratnadhwajpal \\
\hline
\end{tabular}
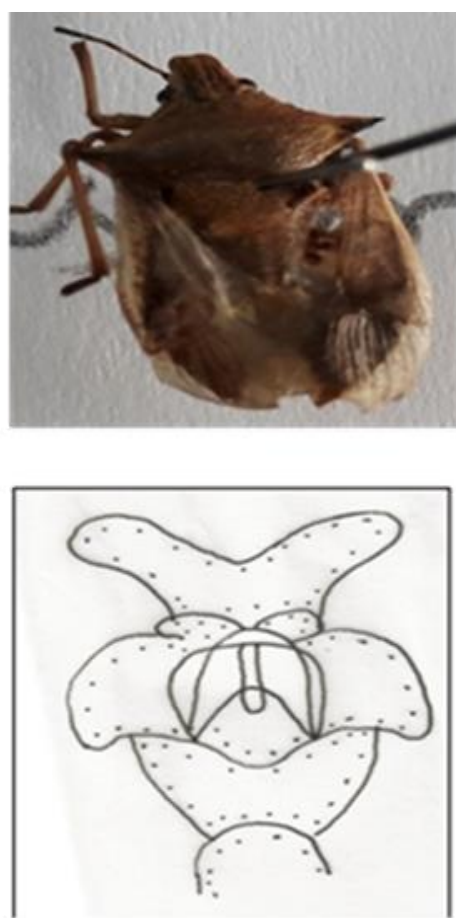

C

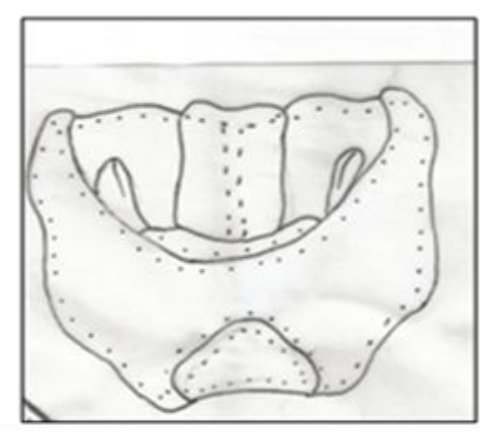

A

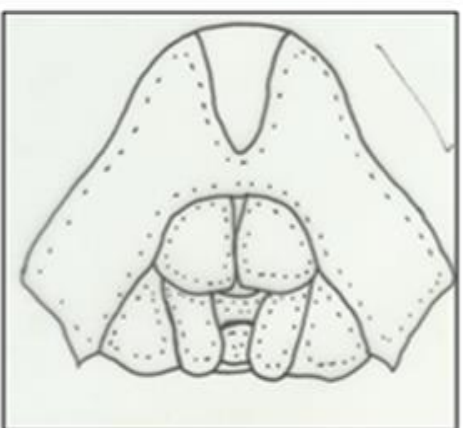

D

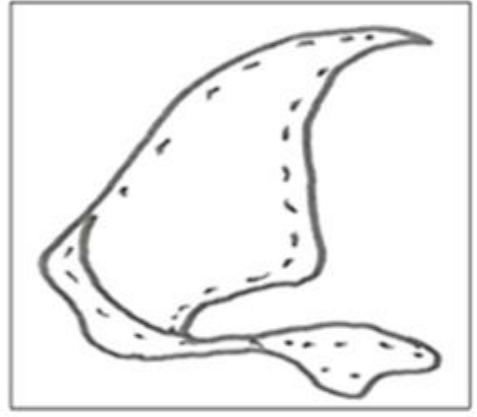

B

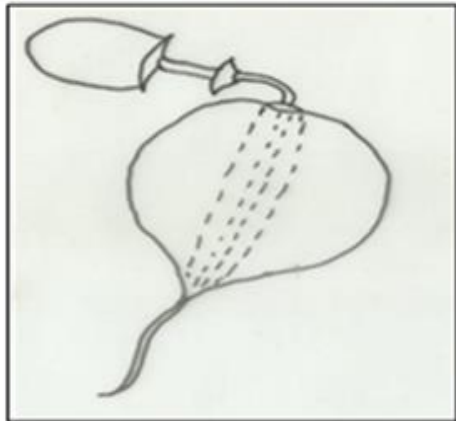

E

Figure 1. (A-E); A; Pygophore 2mm B. Aedeagus: 3mm C.Paramare: $2 \mathrm{~mm}$ D. Terminalia 4mm E. Spermatheca : $2 \mathrm{~mm}$ 


\section{Conclusion}

Genus Canthecona Amyot et Serville first time reported from the district Khairpur Sind Pakistan from the larva lepidopterous, Hymenoptera on grass, herbs and shrubs

\section{Authors' contributions}

Conceived and designed the experiments: $\mathrm{S}$ Mangi \& WA Panhwar, Performed the experiments: S Mangi, Analyzed the data: AM Shaikh \& M Shah, Contributed materials/ analysis/ tools: S Mangi \& W Khan, Wrote the paper: S Mangi \& WA Panhwar,

\section{Acknowledgment}

The authors are highly tankful to the reviewers for the valuable comments.

\section{References.}

1. De Clercq (2008). Predatory Stink Bugs (Hemiptera: Pentatomidae, Asopinae). In: Capinera JL (ed) Encyclopedia of insects, 2nd Ed. Kluwer Acad Publ Dordrecht 30: 42-3045.

2. De Clercq (2000). Predaceous stinkbugs (Pentatomidae: Asopinae), In: Schaefer C.W., Panizzi A.R., (Eds.), Heteroptera of Economic Importance, CRC Press, Boca Raton, London New York Washington D.C.

3. Rolston LH, McDonald FJD \& Thomas DB (1980). A conspectus of Pentatomini genera of the Western Hemisphere. Part I (Hemiptera: Pentatomidae). Jour N Y Entomol Soc 88: $120-132$.

4. Rolston LH (1981). Ochlerini, a new tribe in Discocephalinae (Hemiptera: Pentatomidae). Jour N Y Entomol Soc 89: $40-42$

5. Rolston LH (1984). A revision of the genus Priapismus Distant (Hemiptera: Pentatomidae). Jour Kansas Entomol Soc. (87):119-126.

6. Thomas DB (1992). Taxonomic synopsis of the Asopinae Pentatomidae (Heteroptera) of the Western Hemisphere, Monographs 16. The
Thomas Say Foundation, ESA, Lanham, pp. 1-156.

7. Ahmad I \& McPherson JE (1982). Additional information on male and female genitalia of Parabrachymena lariviere and Brochymena Amyot and serville (Hemiptera: Pentatomidae) Ann Entomol Soc Am 91(6): 800-807.

8. Ganguli J, Chandrakar S \& Puri C (2000). Canthecona furcellata a predatory bug on caterpillars of Clostera sp. Insect Environ 6(2): 79-80.

9. Ray SN, Khan, MA \& Tiwari S (2002). Role of egg parasitoids in the natural control of Clostera fulgurita Walker, a serious pest of poplar. Indi Jour of Ecol 29(1): 90-92.

10. Sangha KS \& Sohi AS (2008). Prospective biological control agents for regulation of population of Clostera fulgurita (Walker) on poplar in Punjab. Indi. Jour of Fores 31(1): 95-98.

11. Ganguli J, Chandrakar S \& Puri C (2000). Canthecona furcellata a predatory bug on caterpillars of Clostera sp. Insect Environ 6(2): 79-80.

12. Sangha KS \& Sohi AS (2008). Prospective biological control agents for regulation of population of Clostera fulgurita (Walker) on poplar in Punjab. Indi. Jour of Fores 31(1): 95-98.

13. McPherson JE (1982). The Pentatomidae (Hemiptera) of Northeastern North America. Southern Illinois University Press, Illinois 240.

14. Schaefer CW (1968). The homologies of the female genitalia in the Pentatomidae (Hemiptera: Heteroptera) J ANYE Ento Soc 76(2): 87-91.

15. Afzal \& Ahmad I (1981). A new genus and three new species of Halyini Stal ((Heteroptera: Pentatomidae: Pentatominae). Pak J Zool 3(1\&2): 6371.

16. Ahmad I \& Nazeer R (1988). A revision of the genus Canthecona Amyot et 
serville (Hemiptera, Pentatomidae, Pentatominae, Asopini) from Indo Pak subcontinent. Proc Pak Congr Zool (8): 147-153.

17. Ahmad I (1986). A fool proof technique for inflation of male genitalia in Hemiptera (Insecta) Heteroptera. Pak J Entomol Kar 1(2): 111-112.

18. Schuh RT \& Slater JA (1995). True Bugs of the World (Hemiptera: Heteroptera).
Classification and Natural History. Cornell University Press, Ithaca, New York. xii + pp. 336.

19. Ahmad I \& McPherson JE (1982). Additional information on male and female genitalia of Parabrachymena lariviere and Brochymena Amyot and Serville (Hemiptera: Pentatomidae). Ann Entomol Soc Am 91(6): 800-807. 